\title{
Trends and predictors of rehospitalisation following an acute coronary syndrome: report from the Australian and New Zealand population of the Global Registry of Acute Coronary Events (GRACE)
}

\author{
Prashanthi V Sangu, ${ }^{1}$ Isuru Ranasinghe, ${ }^{1}$ Bernadette Aliprandi Costa, ${ }^{1}$ Gerard Devlin, ${ }^{2}$ \\ John Elliot, ${ }^{3}$ Jeffery Lefkovitz, ${ }^{4}$ David Brieger ${ }^{1}$
}

'Department of Cardiology, Concord Repatriation General Hospital, Concord, New South Wales, Australia

2Department of Cardiology, Waikato Hospital, Hamilton, New Zealand

${ }^{3}$ Department of Cardiology, Christchurch Hospital,

Christchurch, New Zealand ${ }^{4}$ Department of Cardiology, Royal Melbourne Hospital, Victoria, Australia

\section{Correspondence to} Prof. David Brieger, Department of Cardiology, Concord Repatriation General Hospital, Hospital Road, Concord, NSW 2139, Australia; davidb@email.cs.nsw.gov.au

Accepted 28 August 2012 Published Online First 25 September 2012

\begin{abstract}
Background Readmission following an acute coronary syndrome (ACS) is frequent in our community. Patient specific factors identifying those at risk of readmission are poorly described.
\end{abstract}

Methods Data were analysed from 5219 patients with an ACS enrolled in the Australian and New Zealand population of the Global Registry of Acute Coronary Events (GRACE) between 1999 and 2007. Patients who were readmitted for cardiovascular disease within 6 months of discharge were identified; regression analysis was used to predict independent patient factors associated with readmission 1 month and 1-6 months after discharge.

Results 1048 patients (20.1\%) were readmitted within 6 months, with a significant proportion $(n=434,41.4 \%)$ of readmissions occurring within 30 days of discharge. Readmission within 6 months was associated with a higher incidence of unscheduled cardiac catheterisation (HR 25.64, 95\% Cl 18.41 to 35.71), unscheduled percutaneous coronary intervention (PCI) (HR 15.78, 95\% $\mathrm{Cl} 10.56$ to 23.59), stroke (HR 1.92, 95\% Cl 1.08 to 3.43), and death (HR 2.40,95\% Cl 1.66 to 3.49). Recurrent ischemia in hospital and a diagnosis of S-T elevation myocardial infarction during the index admission were associated with the strongest risk of early rehospitalisation, while revascularisation by $\mathrm{PCI}$ or coronary artery bypass surgery (CABG) was associated with lowest risk of early readmission. A history of heart failure, prior myocardial infarction or angina was associated with a greater likelihood of later rehospitalisation, whereas revascularisation by CABG was associated with the lowest risk of later rehospitalisation. Conclusions Several patient and clinical factors identify patients at higher risk of readmission. Identifying these factors and escalating in-hospital and post-discharge care for these higher risk patients may prevent readmission and improve outcome.

\section{INTRODUCTION}

An acute coronary syndrome (ACS) is well recognised as a major cause of death and disability and contributes to approximately 95000 hospitalisations annually in Australia. ${ }^{1}$ ACS events carry a high economic burden, with cardiovascular disease related healthcare cost amounting to $\$ 5.5$ million per year; $50 \%$ of that amount is spent on care received during hospitalisation. ${ }^{2}$
Among those who survive an ACS event, rehospitalisation is a common, costly, and often preventable outcome. $^{3-5}$ US data suggest that $13.4 \%$ of acute myocardial infarction (AMI) admissions were followed by a readmission within 15 days, accounting for 21000 admissions at a cost of $\$ 136$ million. ${ }^{6}$ While late (>30 day) readmissions have been attributed to disease specific risk factors, early readmission following index hospitalisation is also widely regarded as reflective of quality of care received. ${ }^{6}$ This is particularly relevant in the context of progressive declining length of stay following hospitalisation and increased pressure for early discharge of patients seen in Australia. ${ }^{1}$

The Global Registry of Acute Coronary Events (GRACE) is a large multinational ACS registry. ${ }^{7}$ The Australia and New Zealand (ANZ) population of this registry was collected over nearly a decade and represents the largest ACS registry population in ANZ. We have previously shown improved utilisation of evidence based therapies, improvement in patient outcome and a reduction in readmission at 6 months following an ACS event in this population over nearly a decade. ${ }^{8}$ We sought to characterise patients who were readmitted following an ACS event, and to evaluate early ( $\leq 30$ day) versus later readmission (30 days to 6 months) following ACS admission and to assess patient characteristic that predicted early and late readmission in the ANZ population.

\section{METHODS}

\section{Study population}

Full details of the GRACE method have been published previously. ${ }^{7}$ GRACE is designed to reflect an unbiased population of patients with ACS. Patients enrolled in the GRACE registry were at least 18 years of age, and presented with symptoms suggestive of coronary ischaemia. In addition to symptoms, patients were required to have either ECG changes consistent with ACS, elevation of serum cardiac biomarkers of myocardial necrosis or documented coronary artery disease. ACS precipitated by non-cardiovascular co-morbidity such as anaemia or trauma was excluded. To enrol an unselected population, the first 10-20 consecutive eligible patients were recruited from each site per month. Data were collected by trained coordinators using standardised case report forms. 
Demographic characteristics, medical history, presenting symptoms, biochemical and electrocardiographic findings, treatment practices, and a variety of hospital outcome data were collected. Selected study outcomes were assessed at 6 months postdischarge. These included unscheduled readmission rates, unscheduled procedures (coronary angiography, percutaneous coronary intervention (PCI), coronary artery bypass surgery $(\mathrm{CABG}))$ and 6 month complications.

Standardised definitions for all patient-related variables and clinical diagnoses were used. For this analysis we limited our study to the ANZ population enrolled in the GRACE registry between 1999 and 2007. We included all patients with a discharge diagnosis of confirmed ACS. ${ }^{7}$ We included patients who directly presented to hospital as well as patients transferred from an outlying hospital.

\section{Statistical analysis}

Data are summarised as frequencies and percentages for categorical variables. Continuous variables are presented as mean and standard deviation if normally distributed; otherwise data are presented as medians and 25th and 75th percentiles. For comparative evaluation of temporal trends, we divided the period of enrolment into 3-yearly groups (1999-2001, 2002-2004 and 2005-2007). Patients were also stratified according to their level of risk at presentation (low, intermediate and high risk) according to the GRACE risk model for in-hospital death using previously published cut-off values. ${ }^{9}$

The $\log$ rank $\chi^{2}$ statistic was used to evaluate for difference between risk groups for each year group. We further evaluated the temporal trend (increase or decrease) in the outcome variable across year groups for each risk category. The double sided Cochran-Armitage test for trend or logistic regression was used to evaluate temporal trends. Stepwise logistic regression analysis was performed to evaluate for independent predictors of readmission. All univariate predicators of readmission with $p$ values of $<0.10$ were included in the regression analysis. Adjusted OR and $95 \%$ CIs were reported for the independent predictors of readmission and outcome associated readmissions. The adequacy of the regression model was assessed with the Hosmer-Lemeshow goodness-of-fit test. A $p$ value of $<0.05$ was used as a cut-off for statistical significance. The analysis was performed using SAS V.9.1. Data were $>98 \%$ complete for all variables, with the exception of referral for cardiac rehabilitation where complete data are only available from year 2000 onwards.

\section{RESULTS}

A total of 5615 patients with a confirmed diagnosis of ACS (S-T elevation myocardial infarction (STEMI), non-STEMI or unstable angina pectoris) were enrolled into the study from 11 hospitals in ANZ, of whom 5219 patients were discharged alive from the index hospitalisation. Of these patients, 1048 $(20.1 \%)$ were readmitted within 6 months of discharge from index hospitalisation, with 434 readmissions (41.4\%) occurring with the first month and the remaining $634(58.6 \%)$ occurring at 2-6 months. Over the 9-year enrolment period, overall rehospitalisation rates declined from $23.7 \%$ to $15.6 \%$ ( $p$ for trend $<0.001$ ) (figure 1). This decline was observed in both early rehospitalisation ( $p$ for trend 0.002 ) and in later rehospitalisation ( $p$ for trend $<0.001$ ).

\section{Baseline characteristics}

The baseline characteristics of readmitted patients and those who were not readmitted are shown in table 1 . Patients who

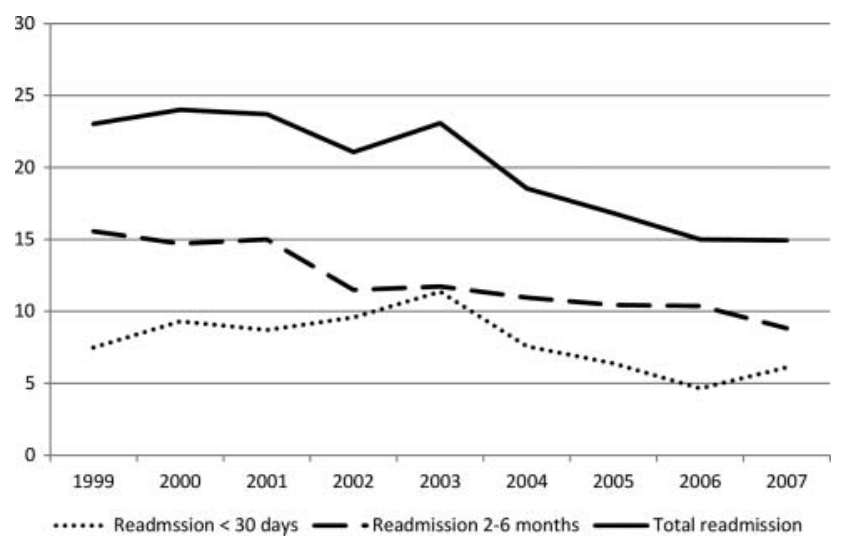

Figure 1 Rates of rehospitalisation post-discharge, 1999-2007. For comparative evaluation of temporal trends the period of enrolment was divided into 3-yearly groups (1999-2001, 2002-2004 and 2005-2007). $p$ Value for change in total readmission $(p<0.001)$, early readmission $(p=0.002)$ and late readmission $(p<0.001)$.

were readmitted were older, more likely to be male, and had a greater burden of cardiac risk factors and prior cardiac disease at index hospitalisation. Readmitted patients were more likely to have had evidence of heart failure (as indicated by the higher Killip class) and were generally at higher risk of further events at index hospitalisation as indicated by the GRACE risk score (all $\mathrm{p}<0.001$ ).

Table 1 Baseline characteristics

\begin{tabular}{|c|c|c|c|}
\hline Characteristic & $\begin{array}{l}\text { Readmission } \\
\text { group, \% }\end{array}$ & $\begin{array}{l}\text { No readmission } \\
\text { group, } \%\end{array}$ & p Value \\
\hline \multicolumn{4}{|l|}{ Demographic } \\
\hline Number & 1048 & 4081 & \\
\hline Age & 67.8 & 65.7 & $<.0001$ \\
\hline Males & 64.0 & 35.7 & 0.001 \\
\hline \multicolumn{4}{|l|}{ Past medical history } \\
\hline AMl & 44.1 & 32.1 & $<0.0001$ \\
\hline Angina & 63.2 & 49.9 & $<0.001$ \\
\hline $\mathrm{CHF}$ & 16.7 & 8.5 & $<0.001$ \\
\hline $\mathrm{PCl}$ & 19.0 & 14.9 & 0.001 \\
\hline CABG & 20.0 & 15.3 & 0.0002 \\
\hline Diabetes & 30.4 & 24.0 & $<0.001$ \\
\hline Hypertension & 64.0 & 55.2 & $<0.001$ \\
\hline Elevated lipids & 59.3 & 55.2 & 0.016 \\
\hline Smoking & 61.4 & 63.6 & 0.19 \\
\hline \multicolumn{4}{|l|}{ Presentation on arrival } \\
\hline $\begin{array}{l}\text { Mean GRACE risk score } \\
\text { on arrival }\end{array}$ & 131.7 & 124.6 & $<0.0001$ \\
\hline Killip class $>1$ & 25.6 & 17.0 & $<.0001$ \\
\hline $\begin{array}{l}\text { Elevated cardiac } \\
\text { enzymes }\end{array}$ & 64.9 & 68.0 & 0.06 \\
\hline S-T changes & 47.2 & 45.0 & 0.19 \\
\hline \multicolumn{4}{|l|}{ Diagnosis } \\
\hline STEMI & 28.1 & 30.5 & 0.12 \\
\hline NSTEMI & 36.6 & 32.1 & \\
\hline Unstable angina & 35.3 & 32.1 & \\
\hline \multicolumn{4}{|l|}{ Enrolment year } \\
\hline 1999-2001 & 23.7 & 76.7 & $<0.0001$ \\
\hline 2002-2004 & 21.0 & 79.0 & \\
\hline 2005-2007 & 15.6 & 84.4 & \\
\hline 2005-2007 & 15.6 & 84.4 & \\
\hline
\end{tabular}

$\mathrm{AMI}$, acute myocardial infarction; $\mathrm{CABG}$, coronary artery bypass graft; $\mathrm{CHF}$, congestive heart failure; NSTEMI, non S-T elevation myocardial infarction; PCI, percutaneous coronary angiography; STEMI, S-T elevation myocardial infarction. 
Table 2 Outcome among readmitted patients

\begin{tabular}{lcc}
\hline & HR & $\mathbf{9 5 \%}$ Cl \\
\hline 6-month death & 2.40 & 1.66 to 3.49 \\
Stroke & 1.93 & 1.08 to 3.43 \\
Unscheduled coronary angiogram & 25.63 & 18.41 to 35.71 \\
Unscheduled percutaneous Coronary intervention & 15.78 & 10.56 to 23.59 \\
\hline
\end{tabular}

\section{Six-month events associated with readmission (table 2)}

Readmission following discharge from index hospitalisation was associated with a marked increased incidence of unscheduled cardiac catheterisation (HR 25.64, 95\% CI 18.41 to 35.71 ) and unscheduled PCI (HR 15.78, 95\% CI 10.56 to 23.59). Additionally, readmission at any time post-discharge was associated with adverse patient outcome at 6 months, including increased risk of stroke (HR 1.93, 95\% CI 1.08 to 3.43) and death (HR 2.40, 95\% CI 1.66 to 3.49) following adjustment for baseline clinical characteristics and differences in treatment and management at index hospitalisation (table 2). The possibility that the clinical threshold for readmission changed during the study period was explored by comparing the prognostic impact of readmission between patients enrolled in the first 2 years (1999-2000) with those enrolled in the last 2 years (2006-2007). Although these data are limited by small numbers, there was no statistically significant difference in likelihood of death, stroke or unscheduled PCI when the first 2 years were compared to the last 2 years as reflected by overlap of CIs in all of measures (table 3).

\section{Independent predictors of early rehospitalisation ( $<\mathbf{3 0}$ days after index ACS admission)}

Female gender, background history of hyperlipidaemia at index hospitalisation, in-hospital prescription of calcium channel antagonists, recurrent ischaemia in hospital, a diagnosis of STEMI and high risk status by the GRACE risk score were associated with a higher risk of first rehospitalisation within 30 days of discharge from the index ACS admission (table 4). In contrast, revascularisation (PCI or CABG), echocardiography in hospital, and admission during the most recent year group (2005-2007) were associated with a lower risk of rehospitalisation within the first 30 days of discharge from the index admission.

\section{Independent predictors of late rehospitalisation (2-6 months after index ACS admission)}

Patients with a greater burden of cardiac disease at index presentation, including prior history of AMI, angina and hypertension were at increased risk of late rehospitalisation (table 5). Prior history of heart failure (OR 1.69, 95\% CI 1.30 to 2.19) and clinical evidence of heart failure at index admission (as assessed by Killip class >1) (OR 1.31, 95\% CI 1.05 to 1.63) were strongly associated with increased risk of late rehospitalisation. Higher diastolic blood pressure at index presentation

Table 3 Prognostic impact of readmission during the study period

\begin{tabular}{lcc}
\hline 6-month outcome & $\mathbf{1 9 9 9 - 2 0 0 0 , ~ H R ~ ( 9 5 \% ~ C l ) ~}$ & $\mathbf{2 0 0 6 - 2 0 0 7 , ~ H R ~ ( 9 5 \% ~ C I ) ~}$ \\
\hline Death & $1.85(0.97$ to 3.52$)$ & $5.22(2.44$ to 11.2$)$ \\
Stroke & $4.25(1.44$ to 12.5$)$ & $2.02(0.2$ to 19.16$)$ \\
Unscheduled PCl & $13.05(6.34$ to 26.8$)$ & $13.6(5.45$ to 33.8$)$ \\
\hline
\end{tabular}

The referent group for all HR indicated in the table is the patients who did not experience a readmission during the same time period.

$\mathrm{PCl}$, percutaneous coronary angiography.
Table 4 Independent predictors of early rehospitalisation (first hospitalisation within 30 days)

\begin{tabular}{ll}
\hline & Odds ratio (95\% Cl) \\
\hline Predictor & \\
Female sex & $1.33(1.062$ to 1.67$)$ \\
History of hyperlipidaemia & $1.67(1.32$ to 2.11$)$ \\
In-hospital PCI & $0.65(0.48$ to 0.87$)$ \\
In-hospital CABG & $0.35(0.17$ to 0.69$)$ \\
In-hospital echocardiography & $0.72(0.56$ to 0.91$)$ \\
In-hospital use of Ca channel blocker & $1.41(1.12$ to 1.79$)$ \\
Recurrent ischemia in hospital & $1.69(1.36$ to 2.11$)$ \\
Discharge diagnosis & \\
UAP & 1.00 \\
NSTEMI & $1.28(0.97$ to 1.70$)$ \\
STEMI & $1.76(1.29$ to 2.40$)$ \\
GRACE risk score & \\
Low risk & 1.00 \\
Intermediate & $1.07(0.82$ to 1.39$)$ \\
High risk & $1.65(1.17$ to 2.31$)$ \\
Year of admission & \\
1999-2001 & 1.00 \\
2002-2004 & $1.32(1.03$ to 1.69$)$ \\
2005-2007 & $0.65(0.48$ to 0.88$)$ \\
\hline
\end{tabular}

CABG, coronary artery bypass graft; NSTEMI, non S-T elevation myocardial infarction; $\mathrm{PCl}$, percutaneous coronary angiography; STEMI, S-T elevation myocardial infarction; UAP, unstable angina pectoris.

and more recent year of enrolment were associated with a lower risk of late rehospitalisation. Revascularisation by CABG was associated with the lowest risk of later rehospitalisation (OR 0.43 , 95\% CI 0.25 to 0.75 ).

\section{DISCUSSION}

Our study suggests that rehospitalisation for heart disease following an ACS remains a significant problem in the ANZ population. While both early and late rehospitalisation promisingly declined over the 9-year study period, even in the most recent year group, $15.6 \%$ of patients (ie, nearly 1 in 6 patients) required rehospitalisation within 6 months of discharge, with more than a third occurring within 30 days. The high associated rates of unscheduled procedures and adverse outcome suggests a persisting burden on both patients and health service providers.

Early rehospitalisation within 30 days of discharge is potentially preventable. As such it is widely regarded as measure of

Table 5 Independent predictors of late rehospitalisation (first hospitalisation with 2-6 months)

\begin{tabular}{ll}
\hline Predictor & Odds ratio (95\% CI) \\
\hline $\begin{array}{l}\text { Prior history of cardiac disease at index hospitalisation } \\
\text { History of AMI }\end{array}$ & $1.27(1.04$ to 1.54$)$ \\
History of CHF & $1.69(1.30$ to 2.19$)$ \\
History of angina & $1.39(1.14$ to 1.71$)$ \\
History of hypertension & $1.24(1.02$ to 1.50$)$ \\
Killip class >1 at index presentation & $1.31(1.05$ to 1.63$)$ \\
In-hospital CABG & $0.43(0.25$ to 0.75$)$ \\
Diastolic blood pressure (per $10 \mathrm{~mm} \mathrm{Hg}$ increase) & $0.94(0.89$ to 0.99$)$ \\
Year of admission & \\
1999-2001 & 1.00 \\
2002-2004 & $0.59(0.47$ to 0.74$)$ \\
$2005-2007$ & $0.71(0.57$ to 0.87$)$
\end{tabular}

AMI, acute myocardial infarction; CABG, coronary artery bypass graft; $\mathrm{CHF}$, congestive heart failure. 
quality care and often reported as a hospital performance measure. Early readmission rates reported in the literature vary substantially due to the heterogeneity in study types and reported outcomes measure. Accepting this limitation however, the 30 -day readmission rate of $5.66 \%$ reported in our study in the most recent enrolment period is substantially lower than the $11.3-28.1 \%$ 30-day readmission rate reported in systematic reviews of rehospitalisation. ${ }^{6}$ While this may suggest comparatively better quality of care within the hospitals included in this analysis, there remains the potential for further reduction in early rehospitalisation. Recurrent ischaemia in hospital and presentation with STEMI were strongly associated with rehospitalisation, whereas revascularisation (by PCI or CABG) was associated with reduced early rehospitalisation. This suggests that better detection and aggressive treatment of underlying cardiac ischaemia (by revascularisation where appropriate) may potentially prevent early rehospitalisation. This is in keeping with a substantial amount of literature which suggests improved outcome for patients with significant ischaemia who are revascularised following an ACS event. The association between female sex and increased risk of early rehospitalisation may also be related to underlying ischaemia. More common atypical and complex ACS presentations and a more conservative approach to revascularisation among women are well documented in the ACS literature. ${ }^{10}$

In contrast to early rehospitalisation, late rehospitalisation (>30 days) is generally attributed to disease specific risk factors. In our analysis, past history of AMI, hypertension, angina and heart failure prior to index hospitalisation (or at presentation) were all associated with increased risk of late rehospitalisation. While late readmissions may seem less preventable, it is important to note that they form nearly two-thirds of all readmissions. There are two broad ranges of interventions proven to reduce readmission in this cohort. Cardiac rehabilitation is well established in reducing mortality, revascularisation and readmission following an AMI. ${ }^{11}{ }^{12}$ Specialised heart failure programmes, including dedicated heart failure clinics and heart failure home visits programmes, are known to reduce readmission and cost, and improve outcome among heart failure patients. However, contemporary data from Australia suggest that only approximately $50 \%$ of patients are referred to cardiac rehabilitation on discharge. ${ }^{8}$ Furthermore, only $6.5 \%$ of hospitals in Australia have heart failure management programmes, with many not adhering to expert management guidelines, ${ }^{13}$ suggesting that these preventative interventions are significantly underutilised.

Our analysis suggests that both early and late rehospitalisation have declined over the 9-year enrolment period, even when adjusted for differences in patient presentation and management. We have previously shown that the presentation risk (as measured by the GRACE risk score) remained unchanged in this population over this enrolment period. ${ }^{8}$ Furthermore, the lack of difference in prognostic impact of readmission over the study period suggests that a higher threshold for readmission was not a factor, and the observed decline in readmission is likely to represent improvement in patient management and outcome. While we did not find any association between individual treatments and improved readmission rates (other than for PCI and CABG), it is likely that this observational study was underpowered to detect effects of individual agents on rehospitalisation rates. Furthermore, GRACE did not systematically collect data on post-discharge care, which may have influenced rehospitalisation rates over the enrolment period.

\section{CONCLUSION}

Rehospitalisation for heart disease remains frequent despite advances in management of ACS patients over nearly a decade and is associated with adverse patient outcome. There are clearly identifiably patient characteristics which are associated with a high likelihood of rehospitalisation. Better identification of these characteristics, ensuring equity of access to revascularisation for high risk patients and implementation of interventions proven to reduce rehospitalisation, are strategies to ensure continued improvement in readmission rates in the future.

Acknowledgements The authors would like to thank the following Australia and New Zealand GRACE investigators and coordinators: Karen Beattie, Pauline Cahill, John Counsell, Gerard Devlin, Craig Juergens, Kelly Langdon, Cathy Martin, Marianne Martin, Kelly Paul, Susan Ruane, Anne Silverstone, Nicola Scott and Jonathon Waites.

Contributors PVS and IR contributed to the planning and reporting of the work described in the article. BAC, GD, JE and JL contributed to the conduct and the reporting of the work described in the article. DB contributed to the planning, conduct and the reporting of the work described in the article and is responsible for the overall content.

Funding GRACE is funded by an unrestricted educational grant from Sanofi-Aventis (Paris, France) to the Center for Outcomes Research, University of Massachusetts Medical School (Worcester, Massachusetts). Sanofi-Aventis had no involvement in the collection, analysis and interpretation of data; in the writing of the report; and in the decision to submit the paper for publication.

Competing interests None.

Ethics approval Concord Hospital Ethics Committee.

Provenance and peer review Not commissioned; externally peer reviewed.

\section{REFERENCES}

1. Australian Institute for Health and Welfare. Monitoring acute coronary syndrome using national hospital data: an information paper on trends and issues. Cat. no. CVD 57. Canberra: AlHW, 2011.

2. Access Economics. The economic costs of heart attack and chest pain (Acute Coronary Syndrome). Canberra, 2009 www.accesseconomics.com.au/ publicationsreports.php.

3. Friedman B, Basu J. The rate and cost of hospital readmissions for preventable conditions. Med Care Res Rev 2004;61:225-40.

4. Van Walraven C, Bennett $C$, Jennings $A$, et al. Proportion of hospital readmissions deemed avoidable: a systematic review. CMAJ 2011;183:E391-402.

5. Jencks SF, Williams MV, Coleman EA. Rehospitalizations among patients in the Medicare fee-for-service program. N Engl J Med 2009;360:1418-28.

6. Desai MM, Stauffer BD, Feringa HH, et al. Statistical models and patient predictors of readmission for acute myocardial infarction: a systematic review. Circ Cardiovasc Oual Outcomes 2009;2:500-7.

7. Fox KAA, Eagle KA, Gore JM, et al. The Global Registry of Acute Coronary Events, 1999 to 2009-GRACE. Heart 2010;96:1095-101.

8. Aliprandi-Costa B, Ranasinghe I, Chow V, et al. Management and outcomes of patients with acute coronary syndromes in Australia and New Zealand, 2000-2007. Med J Aust 2011;195:116-21.

9. Granger CB, Goldberg RJ, Dabbous 0 , et al. Predictors of hospital mortality in the global registry of acute coronary events. Arch Intern Med 2003;163:2345-53.

10. Guru V, Fremes SE, Austin PC, et al. Gender differences in outcomes after hospital discharge from coronary artery bypass grafting. Circulation 2006;113:507-16.

11. Taylor RS, Brown A, Ebrahim S, et al. Exercise-based rehabilitation for patients with coronary heart disease: systematic review and meta-analysis of randomized controlled trials. Am J Med 2004;116:682-92.

12. Ades PA. Cardiac rehabilitation and secondary prevention of coronary heart disease. N Engl J Med 2001;345:892-902.

13. Driscoll A, Worrall-Carter L, Hare DL, et al. Evidence-based chronic heart-failure management programmes: reality or myth? BMJ Qual Saf 2011;20:31-7. 